\title{
Effect of orientational restriction on monolayers of hard ellipsoids
}

\author{
Szabolcs Varga ${ }^{*}$ \\ Permanent address: Institute of Physics and Mechatronics, \\ University of Pannonia, PO Box 158, Veszprém, H-8201 Hungary \\ Yuri Martínez-Ratón \\ Grupo Interdisciplinar de Sistemas Complejos (GISC), Departamento de Matemáticas, \\ Escuela Politécnica Superior,Universidad Carlos III de Madrid, \\ Avenida de la Universidad 30, E-28911, Leganés, Madrid, Spain \\ Enrique Velascd \\ Departamento de Física Teórica de la Materia Condensada and Instituto de Ciencia de Materiales Nicolás Cabrera, \\ Universidad Autónoma de Madrid, E-28049 Madrid, Spain \\ Gustavo Bautista-Carbaja $\$$ \\ Departamento de Física, Universidad Autónoma Metropolitana-Iztapalapa, \\ 09340, México, Distrito Federal, México, and Academia de Matemáticas, \\ Universidad Autónoma de la Ciudad de México, 07160, México, D. F., Mexico \\ Gerardo Odriozole \\ Area de Física de Procesos Irreversibles, División de Ciencias Básicas e Ingeniería, \\ Universidad Autónoma Metropolitana-Azcapotzalco, \\ Av. San Pablo 180, 02200 México, D.F., Mexico.
}

(Dated: June 21, 2021)

\begin{abstract}
The effect of out-of-plane orientational freedom on the orientational ordering properties of a monolayer of hard ellipsoids is studied using Parsons-Lee scaling approach and replica exchange Monte Carlo computer simulation. Prolate and oblate ellipsoids exhibit very different ordering properties, namely, the axes of revolution of prolate particles tend to lean out, while those of oblate ones prefer to lean into the confining plane. The driving mechanism of this is that the particles try to maximize the available free area on the confining surface, which can be achieved by minimizing the cross section areas of the particles with the plane. In the lack out-of-plane orientational freedom the monolayer of prolate particles is identical to a two-dimensional hard ellipse system, which undergoes an isotropic-nematic ordering transition with increasing density. With gradually switching on the out-of-plane orientational freedom the prolate particles lean out from the confining plane and a destabilisation of the in-plane isotropic-nematic phase transition is observed. The system of oblate particles behaves oppositely to that of prolates. It corresponds to a two-dimensional system of hard disks in the lack of out-of-plane freedom, while it behaves similar to that of hard ellipses in the freely rotating case.
\end{abstract}

\section{INTRODUCTION}

Phase behavior of non-spherical hard bodies with their centers of mass confined in planar geometry is receiving considerable attention due to the recent development of the preparation of colloidal particles with various shapes and new experimental techniques. Nowadays it is possible to prepare colloids with several geometrical shapes such as cubes, polyhedrons, octopods, ellipsoids and helices [1-6]. The anisotropic colloids can be confined at the interfaces [7-11], between two parallel solid walls

\footnotetext{
*Electronic address: vargasz@almos.uni-pannon.hu

$\dagger$ Electronic address: yuri@math.uc3m.es

$\ddagger$ Electronic address: enrique.velasco@uam.es

$\S$ Electronic address: gustavo.bautista@uacm.edu.mx

๑Electronic address: godriozo@azc.uam.mx
}

$[12,13]$, at the bottom of the sample holder [14], at a substrate surface [15] and into a lamellar matrix of surfactants $[16,17]$. The confinement can be so strong that even colloidal monolayers can be realized experimentally. Ordering properties of two-dimensional and quasi twodimensional (q2D) non- spherical colloids has been the subject of several experimental and theoretical studies [17-36]. The reason for this is that the nature of twodimensional $(2 \mathrm{D})$ nematic ordering is quite different from the three-dimensional (3D) one. It shows only quasi-longrange orientational order with algebraically decaying orientational correlations and the ordering transitions between isotropic and nematic phases are first order or continuous through a Kosterlitz-Thouless disclination unbinding type mechanism [18, 37]. Strictly 2D colloidal systems cannot be examined experimentally, because the out-of- plane orientational and positional freedoms are always present to some extent. Therefore it is desirable to extend the theoretical studies in such directions, 
where the extra orientational and positional freedoms are present. Along this line, the ordering properties of microtubules confined in a thin slit have been modeled as hard spherocylinders placed between two planar walls in [28]. In agreement with the experiment it has been found that the isotropic-nematic transition density increases with the wall separation [28]. Ellipsoidal shaped colloidal particles are gaining widespread applications due to development of the stretching techniques in preparation of monodisperse prolate and oblate ellipsoids from spherical latex particles [4,38]. They can be also confined into planar geometry and create a monolayer to study ordering and glassy behavior of the q2D ellipsoid systems [12,13].

In this paper we examine the orientational ordering properties of q2D hard ellipsoid systems, where particles are allowed to rotate out the confinement plane to some extent, while it is assumed in first approximation that the centers of the particles are always in the same plane. Switching on the out-of-plane orientational freedom by gradual increase (decrease) of the limiting polar angle $\left(\theta_{c}\right)$ for oblate (prolate) shapes, it is possible to make a link between strictly 2D hard ellipse (hard disk) systems for prolate (oblate) shapes and q2D freely rotating prolate (oblate) ellipsoids systems. We show that the additional out-of-plane orientational freedom changes substantially the orientational ordering and the transition properties of both oblate and prolate shaped ellipsoids. To maximize the available free area on the confining surface, the axis of revolution of the prolate particle leans out from the plane, while the oblate particle leans into the plane. As a consequence, the freely rotating prolate ellipsoids resemble hard disks at high densities, while those of oblate ellipsoids behave similarly to hard ellipses. The isotropic-nematic phase transition of hard ellipses corresponds to a planar nematic-biaxial nematic phase transition of the oblate ellipsoids. Here we note that our present work can be considered as an extension of our previous studies done for monolayers of uniaxial and biaxial hard particles using restricted-orientation approximation $[39,40]$.

The paper is organized as follows. The molecular model and the details of the confinement are presented in Sec. II. Sec. III is devoted to the Parsons-Lee theory of q2D hard ellipsoid fluids, where we show how to determine the equilibrium free energy, surface coverage, and order parameters of the uniaxial and biaxial nematic phases. Technical details of the replica exchange Monte Carlo simulation method are given in Sec. IV. The order parameters, the surface coverage, the equation of state and the phase diagram of the system hard ellipsoids are presented in Sec. V. Finally, some conclusions are drawn in Sec. VI.

\section{MOLECULAR MODEL}

In this work we examine the q2D system of hard ellipsoids, where the particles are allowed to rotate freely
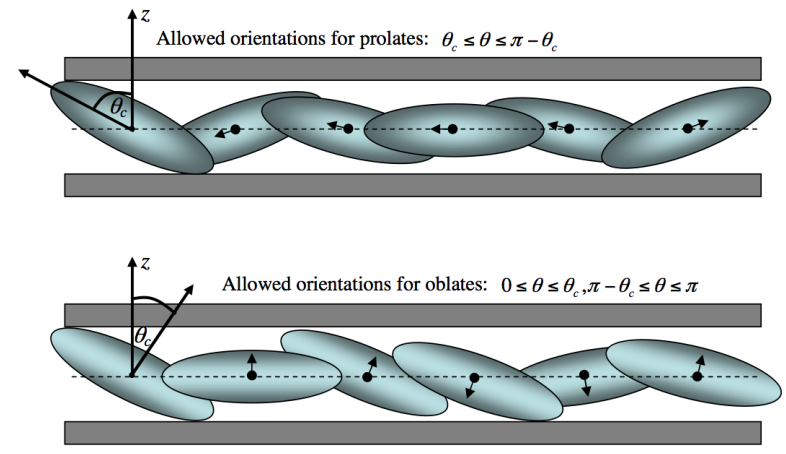

FIG. 1: Schematic representation of the system of confined hard ellipsoids: prolate ellipsoids (upper panel), oblate ellipsoids (lower panel). The particles are confined both positionally and orientationally. The center of mass of the ellipsoid is always in the XY plane, which is denoted by the dashed line, while the ellipsoid can rotate freely in azimuthal angle $(\phi)$ and is restricted by the polar angle $\left(\theta_{c}\right)$. The arrows indicate the direction of the main symmetry axis of the ellipsoids.

in a restricted region of the solid angle, while the centers of particles can move only on a two-dimensional XY plane. The shape of the particles can be both prolate and oblate, i.e. the aspect ratio or shape anisotropy ( $\kappa=\sigma_{\|} / \sigma_{\perp}$, where $\sigma_{\|}$and $\sigma_{\perp}$ are the lengths along and perpendicular to the axis of revolution of the ellipsoid, respectively) can be either larger than one $(\kappa>1$, prolate shape) or between zero and one $(0<\kappa<1$, oblate shape). The orientational restriction takes place only in the polar angle $(\theta)$, which is measured from the axis of revolution of each particle to the normal of the confining XY plane. While the particles can rotate freely in the azimuthal angle $(0<\phi<2 \pi)$, the allowed range of the polar angle is different for prolate and oblate ellipsoids, namely, the range of the polar angle is given by $\theta_{c}<\theta<\pi-\theta_{c}$ for prolate shapes, while $0<\theta<\theta_{c}$ and $\pi-\theta_{c}<\theta<\pi$ intervals are allowed for oblate ones (see Fig. 11. We can tune $\theta_{c}$ between 0 and $\pi / 2$. In the case of prolate ellipsoids $(\kappa>1) \theta_{c}=0$ corresponds to the confined system of freely rotating particles, while $\theta_{c}=\pi / 2$ gives the two-dimensional system of hard ellipses. The situation is different for oblate ellipsoids $(0<\kappa<1)$, because the hard disk limit is given by $\theta_{c}=0$, while the freely rotating system of confined oblate ellipsoids corresponds to $\theta_{c}=\pi / 2$. The interaction between ellipsoids is purely hard, i.e. the pair potential between two particles is given by

$$
u\left(r_{12}, \boldsymbol{\omega}_{12}, \boldsymbol{\omega}_{1}, \boldsymbol{\omega}_{2}\right)=\left\{\begin{array}{lc}
\infty, & \text { for } r_{12} \leq \sigma\left(\boldsymbol{\omega}_{12}, \boldsymbol{\omega}_{1}, \boldsymbol{\omega}_{2}\right) \\
0, & \text { otherwise }
\end{array}\right.
$$

where $r_{12}$ is the center-to-center distance, $\boldsymbol{\omega}_{12}=\left(\cos \phi_{12}, \sin \phi_{12}, 0\right)$ is the unit vector connecting the centers of the two ellipsoids, $\boldsymbol{\omega}_{i}=\left(\sin \theta_{i} \cos \phi_{i}, \sin \theta_{i} \sin \phi_{i}, \cos \theta_{i}\right)$ is the orientational unit vector of particle $i(i=1,2)$ and $\sigma(\cdots)$ is the distance of closest approach. Note that the third 
component of $\boldsymbol{\omega}_{12}$ is always zero, which ensures that the centers of the particles are always in the XY plane. The distance of closest approach between two ellipsoids $[\sigma(\cdots)]$ is approximated in the manner proposed in our previous works $[41,42]$.

\section{PARSONS-LEE THEORY}

To describe theoretically the orientational ordering properties of the monolayer of hard ellipsoids we derive our working equations from the well-known Parsons-Lee (PL) theory of hard bodies [43,44], which proved very successful in determination of the equation of state and the transition properties of IN phase coexistence of nonspherical hard body fluids both in two [23,24] and three dimensions [45-47]. Here we present only the important ingredients of the theory, specific equations for the ellipsoid monolayer and some technical details. It is customary to deal with the free energy of the system, which is the sum of ideal and excess free energy terms, i.e. $F=F_{\text {id }}+F_{\text {ex }}$. The ideal term can be determined exactly from

$$
\frac{\beta F_{\mathrm{id}}}{N}=\log \rho-1+\int d \omega f(\omega) \log f(\omega),
$$

where $\beta=1 / k_{\mathrm{B}} T$ is the inverse temperature, $N$ is the number of particles, $\rho=N / A, A$ is the area of the plane, $\omega=(\phi, \theta)$ is the collection azimuthal and polar angles, $d \omega=d \phi d \theta \sin \theta$ and $f(\omega)$ is the normalized orientational distribution function $\left(\int d \omega f(\omega)=1\right)$. The ranges of the azimuthal and polar angles in the integrations have been already given in Sec. II. The excess free energy contribution can be obtained approximately with the mapping procedure from the actual system to a reference one, where the second virial coefficient and the excess free energy can be obtained with good accuracy. In our case we choose the system of $2 \mathrm{D}$ hard disks as a reference system, because our confined ellipsoid system is $\mathrm{q} 2 \mathrm{D}$. The second virial coefficient of the hard disks is given by $B_{2}^{\mathrm{HD}}=D^{2} \pi / 2$, where $D$ is the diameter of the hard disk. A luckily simple and accurate expression can be derived for the excess free energy of hard disks, too, using the scaled particle theory [48]

$$
\frac{\beta F_{\mathrm{ex}}^{\mathrm{HD}}}{N}=-\ln \left(1-\eta_{\mathrm{HD}}\right)+\frac{\eta_{\mathrm{HD}}}{1-\eta_{\mathrm{HD}}},
$$

where $\eta_{\mathrm{HD}}=\rho a_{\mathrm{HD}}$ is the packing fraction of the hard disk and $a_{\mathrm{HD}}=B_{2}^{\mathrm{HD}} / 2$ is the area of the hard disk. To perform the mapping procedure from the hard ellipsoids into hard disks we introduce the surface coverage (or packing fraction) of the hard ellipsoids, because the intersection of the ellipsoid with the XY plane is an ellipse with characteristic lengths depending on the orientation of the ellipsoid. These characteristic lengths of the ellipse can be calculated easily using simple geometry. One can derive that $\sigma_{\perp, e}=\sigma_{\perp}$ and $\sigma_{\|, e}=$ $\sigma_{\|} \sigma_{\perp} / \sqrt{\sigma_{\perp}^{2} \sin ^{2} \theta+\sigma_{\|}^{2} \cos ^{2} \theta}$, where $\theta$ is the polar angle of the ellipsoid. This means that the monolayer of the hard ellipsoids can be visualized as a multicomponent mixture of hard ellipses on the XY plane. Using the dimensions of the hard ellipse, the intersected area of the hard ellipsoid with the XY plane can be obtained from

$$
a(\theta)=\frac{\pi \sigma_{\perp}^{2} \sigma_{\|}}{4 \sqrt{\sigma_{\perp}^{2} \sin ^{2} \theta+\sigma_{\|}^{2} \cos ^{2} \theta}} .
$$

This polar angle dependent area together with the orientational distribution function allows us to determine the surface coverage of the plane by ellipses, which is given by $\eta=\rho\langle a\rangle$, where the average area of the intersected ellipse can be determined from $\langle a\rangle=\int d \omega a(\theta) f(\omega)$. Note that the surface coverage gives back the packing fraction of hard disks in the hard-sphere limit, because $\sigma_{\|}=\sigma_{\perp}=D$ and the distribution function is a constant. In PL approach the area of hard disks and the average area of hard ellipses are assumed to be the same $\left(a_{\mathrm{HD}}=\langle a\rangle\right)$, which implies also that $\eta_{\mathrm{HD}}=\eta$ due to one-to-one correspondence between the two systems. Based on the concept of Parsons and Lee we can now calculate the excess free energy of the monolayer of hard ellipsoids using the thermodynamic properties of hard disks as follows

$$
\frac{\beta F_{\mathrm{ex}}}{N} \approx \frac{\beta F_{\mathrm{ex}}}{N} \frac{B_{2}^{\mathrm{HE}}}{B_{2}^{\mathrm{HD}}}
$$

where the second virial coefficient of hard ellipsoids restricted to XY plane is given by

$$
B_{2}^{\mathrm{HE}}=\frac{1}{2} \int d \omega_{1} f\left(\omega_{1}\right) \int d \omega_{2} f\left(\omega_{2}\right) A_{\mathrm{excl}}\left(\omega_{1}, \omega_{2}\right) .
$$

This equation contains the excluded area between two ellipsoids, which can be obtained from the distance of closest approach $(\sigma)$ as follows

$$
A_{\mathrm{excl}}\left(\omega_{1}, \omega_{2}\right)=\frac{1}{2} \int_{0}^{2 \pi} d \phi_{12} \sigma^{2}\left(\boldsymbol{\omega}_{1}, \boldsymbol{\omega}_{2}, \boldsymbol{\omega}_{12}\right) .
$$

Using Eqs. (3)-(6) and $\eta_{\mathrm{HD}}=\eta$ condition the excess free energy of the system becomes

$$
\begin{aligned}
& \frac{\beta F_{\mathrm{ex}}}{N}=\left[-\ln (1-\eta)+\frac{\eta}{1-\eta}\right] \\
& \times \frac{\int d \omega_{1} f\left(\omega_{1}\right) \int d \omega_{2} f\left(\omega_{2}\right) A_{\mathrm{excl}}\left(\omega_{1}, \omega_{2}\right)}{4 \int d \omega a(\theta) f(\omega)} .
\end{aligned}
$$

The sum of Eqs. (2) and (8) constitutes our density functional equation to determine the equilibrium orientational distribution function $[f(\omega)]$ and the total free energy density at a given density $(\rho=N / A)$. The minimization of the free energy functional with respect to $f(\omega)$ gives us the Euler-Lagrange equation for the equilibrium $f(\omega)$. Note that the minimization must be carried out by maintaining the normalization condition 
$\left(\int d \omega f(\omega)=1\right)$. Although the minimization procedure is very simple and straightforward, we do not present the equation of $f(\omega)$, since the resulting equation is too long. Once the equilibrium $f(\omega)$ is determined with a standard iterative method, the free energy density can be obtained by the substitution of the resulting $f(\omega)$ into the free energy functional (sum of Eq. (2) and (8)). We determine the pressure from the free energy using $P=\rho^{2} \partial(F / N) / \partial \rho$ and examine the orientational ordering properties of the monolayer by the standard uniaxial and biaxial order parameters. These are defined as

$$
S=\left\langle P_{2}\right\rangle=\int d \omega f(\omega) P_{2}(\theta)
$$

and

$$
\Delta=\int d \omega f(\omega) \sin ^{2} \theta \cos (2 \phi),
$$

where $P_{2}(x)=3 x^{2} / 2-1 / 2$ is the second order Legendre polynomial. The uniaxial order parameter $(S)$ is positive for out-of-plane ordering $(0<S<1)$, while it is negative for in- plane ordering $(-1 / 2<S<0)$. The biaxial order parameter $(\Delta)$ can be very useful in finding in-plane orientational ordering transitions, because $\Delta$ is zero for in-plane complete disorder, while it is nonzero for in-plane order. $S$ is practically a three-dimensional order parameter of bulk ellipsoids, while $\Delta$ is the corresponding two-dimensional one of bulk ellipses when $\theta_{c}=\pi / 2$ . Since our system is q2D, we need both of them. To see the effect of out-of-plane orientational freedom and to which extent the particles are tilted off from the confining plane, we determine the average aspect ratio of the effective ellipse system, which is defined as $\kappa_{\text {eff }}=\left\langle\sigma_{\|, e} / \sigma_{\perp, e}\right\rangle$. In Sec. $V$ we plot the density and the pressure in dimensionless units, which are defined as $\rho^{*}=N \sigma_{\perp}^{2} / A$ and $P^{*}=\beta P \sigma_{\perp}^{2}$.

\section{REPLICA EXCHANGE MONTE CARLO SIMULATION}

As for the 2D hard ellipse study [35], we are implementing the replica exchange Monte Carlo technique [49-51]. This is done to avoid, as far as possible, the inherent hysteresis associated to transitions [52]. The method is based on the definition of an extended ensemble with partition function $Q_{\text {ext }}=\prod_{i=1}^{n_{r}} Q_{i}$, where $Q_{i}$ is the partition function of ensemble $i . n_{r}$ ensembles are considered, and $n_{r}$ replicas are employed to sample the extended ensemble, each one at each ensemble. Defining $Q_{\text {ext }}$ allows introducing swap trial moves between any two replicas, whenever the detailed balance condition is satisfied. In our case it is convenient to expand isobaric-isothermal ensembles in pressure [53]. This is so since we are studying hard particles. Hence, the partition function of the extended ensemble reads $[53,54]$

$$
Q_{\mathrm{ext}}=\prod_{i=1}^{n_{r}} Q_{N T P_{i}}
$$

where $Q_{N T P_{i}}$ is the partition function of the isobaricisothermal ensemble of the system at pressure $P_{i}$ and temperature $T$. $N$ particles are taken into account at each ensemble. A standard implementation is used to sample the NTP i ensembles. This implies independent trial 2D displacements, 3D rotations of single ellipsoids, and area changes of the simulation cell. In case of having confining planes, 3D rotations of single ellipsoids are constrained by their presence. We are also accounting for non-orthogonal parallelogram cells and so, additional trial changes of the angles and relative length sides of the cell lattice vectors are included. The following acceptance rule is set [53]

$$
P_{\mathrm{rm}, \mathrm{acc}}=\min \left\{1, \exp \left[\beta\left(P_{i}-P_{j}\right)\left(A_{i}-A_{j}\right)\right]\right\},
$$

where $A_{i}-A_{j}$ is the area difference between replicas $i$ and $j$. Adjacent pressures must be close to provide swap acceptance rates over 0.1. Simulations are started from a packed triangular arrangement of spheres which are elongated in the direction normal to the plane by a factor $\kappa$. In case of oblates, a stretching factor $\kappa$ is also applied in a certain in-plane direction. Conversely to the stretching of spheres in a 3D cell, this procedure leads to the largest packed arrangement of spheroids in a plane [55]. A stationary state is reached faster by decompressing packed cells than by compressing lose random configurations [52]. We perform the necessary trial moves to observe a stationary state. At this stage we adjust maximum displacements to produce acceptance rates close to 0.3 . We also relocate all pressures, initially set by following a geometric progression with the replica index, to obtain similar swap acceptance rates for all pairs of adjacent ensembles. Next, we perform $4 \times 10^{12}$ sampling trials for fixed maximum particle displacements, maximum rotational displacements, maximum area changes, and maximum changes of the lattice vectors. Verlet neighbor lists [56] are used to improve performance. We set $N \sim 400$ ellipsoids and $n_{r}$ as a function of the pressure range to be covered. $N \sim 400$ is sufficiently large in view of $\mathrm{Xu}$ et. al. analysis of system size effects [33]. More details on the employed methods are given in previous works [52].

\section{RESULTS AND DISCUSSION}

In this section we present our theoretical and simulation results for hard ellipses, freely rotating and orientationally restricted monolayers of hard ellipsoids. The equation of state, the surface coverage, the in-plane and out-of-plane order parameters and the isotropic-nematic (IN) transition densities are determined for various values of shape anisotropy. We start with the system of hard ellipses, which is obtained by setting the polar angles of all ellipsoidal particles $(\theta)$ to be $\pi / 2$. This condition is accompanied by $f(\omega)=f(\phi)$ and that the uniaxial order parameter $(S)$ is always $-1 / 2$ and the biaxial order parameter can be obtained from $\Delta=\int_{0}^{2 \pi} d \phi f(\phi) \cos (2 \phi)$. Note that $\Delta$ now serves as a $2 \mathrm{D}$ orientational order parameter. 

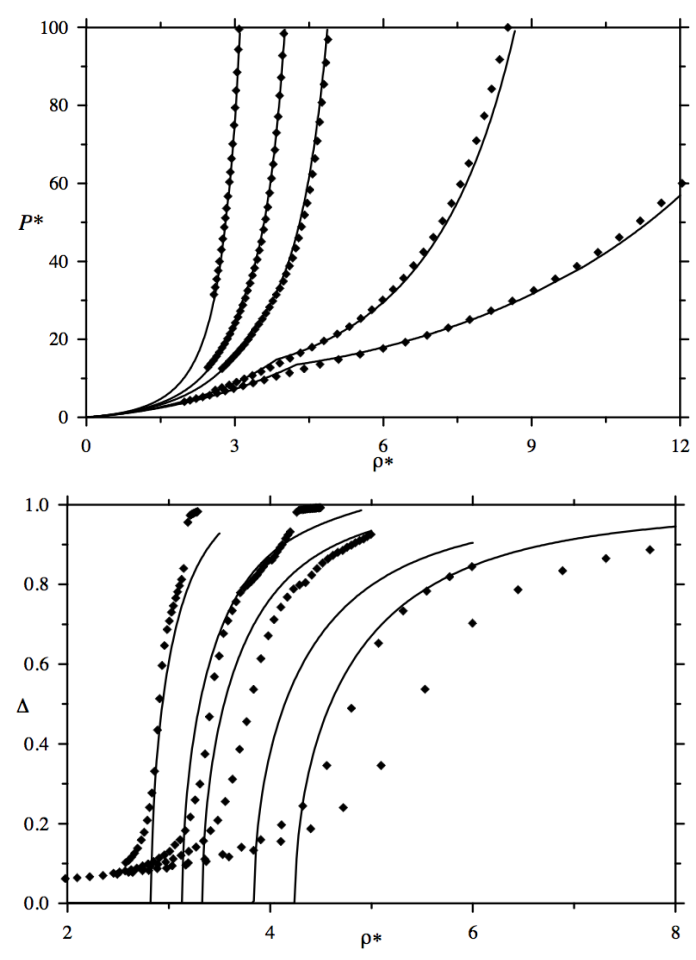

FIG. 2: Orientational ordering of the system of hard ellipses: the equation of state (upper panel) and the two-dimensional order parameter vs. density (lower panel). The curves correspond to $\kappa^{-1}=3,4,5,10$ and 20 from left to right. The curves are the results of PL theory, while the diamond symbols correspond to MC simulation results. The hard ellipse system is obtained from oblate ellipsoids by tilting their orientations into the XY plane.

The theoretical calculations and simulations can also be carried out with both oblate-shaped $(0<\kappa<1)$ and prolate-shaped hard ellipsoids $(\kappa>1)$, where $\theta=\pi / 2$ for all particles. Here we perform the hard ellipse study using oblate-shaped ellipsoids. Fig. 2 shows the pressure and the 2D order parameter $(\Delta)$ as a function of density for $\kappa^{-1}=3,4,5,10$ and 20 . One can see that more particles on the surface (higher densities) are required to maintain the same value of the pressure with increasing shape anisotropy. This is due to the fact that the XY plane becomes more spacious with increasing $\kappa^{-1}$, i.e. $\sigma_{\|}$must decrease at fixed $\sigma_{\perp}$.

It can be also seen in Fig. 2 that the simulation data are well reproduced by the approximate PL theory except the values of the order parameters of the $\mathrm{N}$ phases corresponding to extremely anisotropic particles $\left(\kappa^{-1}=10\right.$ and 20). These data well agree with those given in [33]. The order parameter curves reveal for the occurrence of isotropic-nematic phase transition, because the phase is isotropic at low densities $(\Delta=0)$, while it is nematic at high ones $(\Delta>0)$. The phase transition is second order in the theory, while it is higher order (KosterlitzThouless type continuous transition) in the simulation.

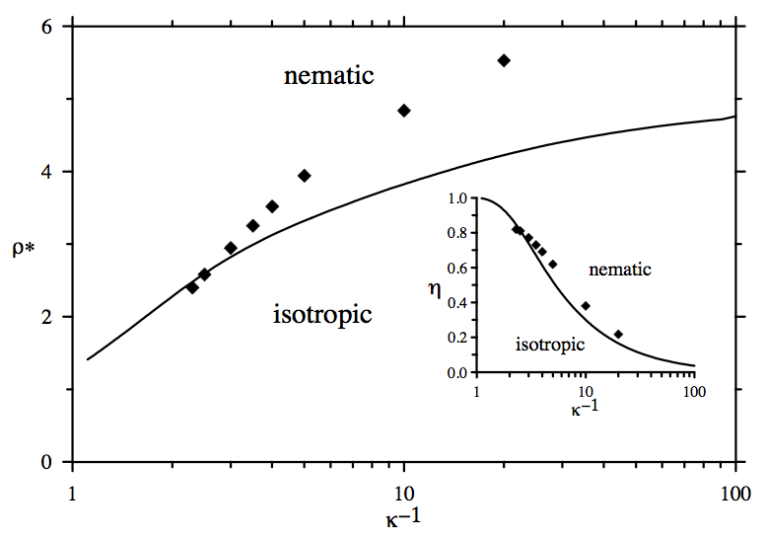

FIG. 3: Shape dependence of the isotropic-nematic (IN) transition of hard ellipses: reduced density vs. aspect ratio. The curves are the results of PL theory, while the diamond symbols correspond to MC simulation results. The inset shows the packing fraction of the IN transition as a function of aspect ratio, where $\eta=\rho \pi \sigma_{\|} \sigma_{\perp} / 4$.

Both the theory and the simulation show the same tendencies for IN transition densities and packing fractions (see Fig. 3). Making the ellipsoids more anisotropic one can see that the IN transition density increases, while the IN packing fraction decreases. This apparent contradiction is due to the fact that the increasing shape anisotropy makes the system more spacious $\left(\sigma_{\perp}\right.$ is constant, while $\sigma_{\|}$is decreasing), which requires more particles for the initiation of the phase transition, while the $\mathrm{XY}$ plane can be less occupied at the same time. It can be also seen that the theory underestimates IN transition densities and packing fractions in higher extent with increasing shape anisotropy, which is due to the fact that the contribution of higher order virial coefficients are not negligible with increasing $\kappa^{-1}$.

The monolayer of freely rotating hard ellipsoids can be achieved by using $\theta_{c}=\pi / 2$ and $\theta_{c}=0$ limiting angles for oblate and prolate shapes, respectively. The resulting surface coverage, effective aspect ratio, pressure and order parameters are shown for prolate and oblate shaped ellipsoids in Figs. 4 and 5 respectively. In these figures the effective aspect ratio of the corresponding hard ellipse system is given by $\kappa_{\text {eff }}=\left\langle\sigma_{\|, e} / \sigma_{\perp, e}\right\rangle=$ $\left\langle\kappa / \sqrt{\sin ^{2} \theta+\kappa^{2} \cos ^{2} \theta}\right\rangle$, which is the average aspect ratio of the ellipses obtained by intersecting the ellipsoids by the XY plane. In turn, the surface coverage can be obtained with the help of $\kappa_{\text {eff }}$ as follows: $\eta=\rho^{*} \pi \kappa_{\text {eff }} / 4$. Starting with prolates ellipsoids, one can see that the particles do not form an isotropic phase, but they align along the normal of the XY plane (see the simulation snapshot of Fig. (4) even at very low densities. In fact, an isotropic phase would appear only at the limit of infinite dilution. The density dependence of the surface coverage and the effective aspect ratio shows that the particles have less and less chance to lie into the XY surface with increas- 

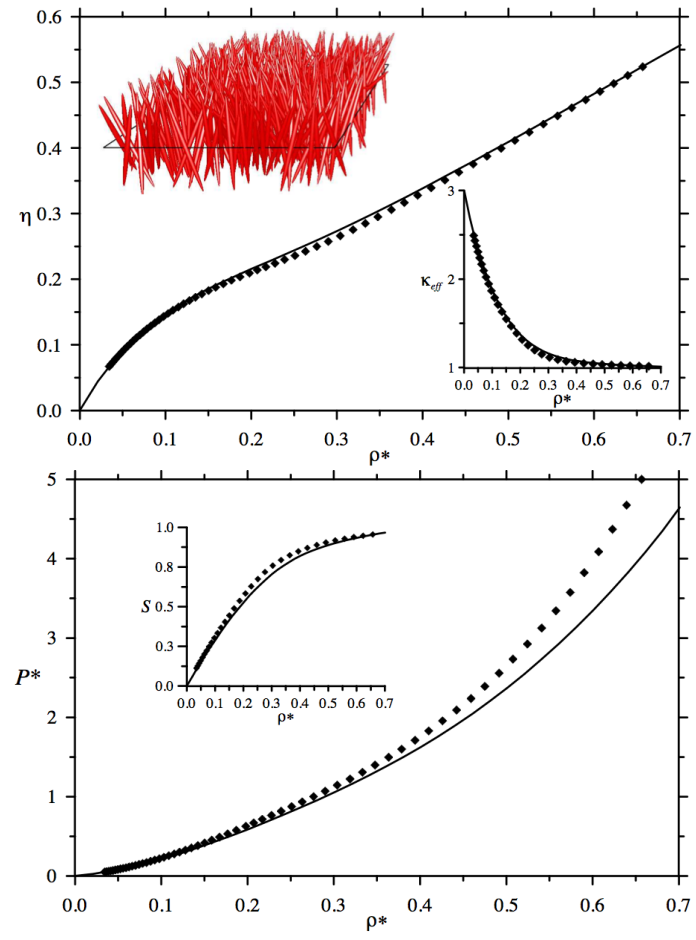

FIG. 4: Monolayer of freely rotating hard prolate ellipsoids: surface coverage (packing fraction) vs. reduced density (upper panel) and the equation of state (lower panel) at $\kappa=10$. The effective aspect ratio of the corresponding hard ellipse system $\left(\kappa=\left\langle\sigma_{\|, e} / \sigma_{\perp, e}\right\rangle\right)$ is shown in the inset of the upper panel, while the out-of-plane order parameter $(S)$ is presented in the inset of the lower panel. The curves are the results of PL theory, while the diamond symbols correspond to MC simulation results. The snapshot is the result of replica exchange Monte Carlo simulation method.

ing density because of packing effects. The intersection of the ellipsoid with the XY surface is practically a hard disk for $\rho^{*}>0.6$, where the effective ellipse aspect ratio is almost one. This makes the monolayer of hard ellipsoids very similar to the system of hard disks at high densities despite the presence of orientational fluctuations. The inplane order parameter (Eq. 10) is always zero, while the out-of-plane order parameter (Eq. 9) is positive. This shows that the out-of-plane orientational ordering is uniaxial with nematic director parallel to the layer normal. It can be also seen that $S$ is very close to its maximum value $\left(S_{\max }=1\right)$ at $\rho^{*}=0.7$, which corresponds to the case where all prolate ellipsoids are parallel and align along the layer normal. The reason why prolate ellipsoids prefer the out-of-plane ordering is that they can maximize the free area available on the surface with the penalty of orientational entropy loss. Moreover the close packing structure of prolate ellipsoids is identical to that of $2 \mathrm{D}$ hard disks. One can also see that the theory reproduces quite well the simulation data for all properties except the equation of state at high densities. Regarding the possible freezing transition at high densities, it has
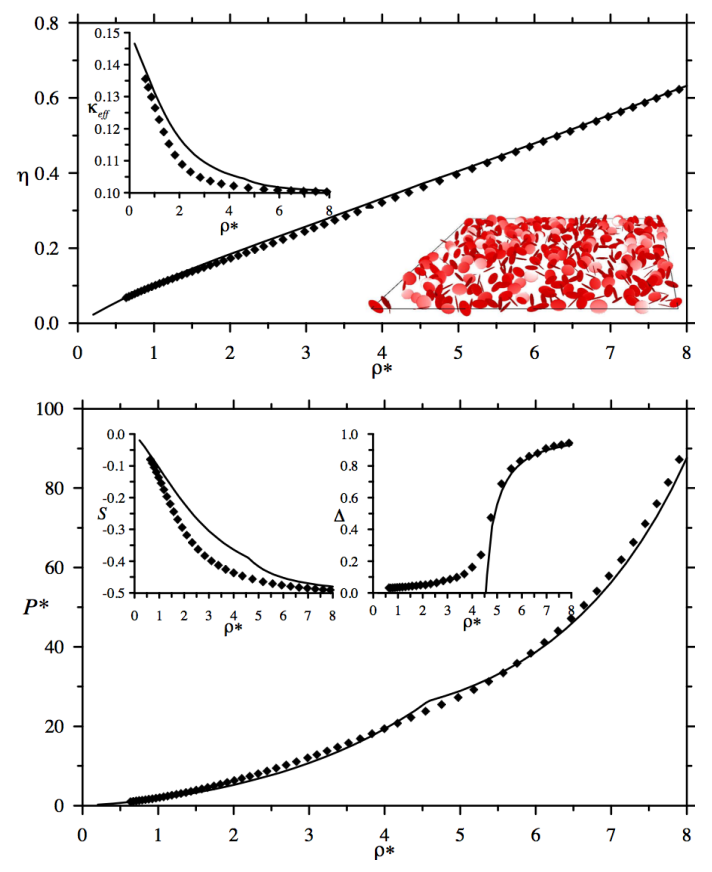

FIG. 5: Monolayer of freely rotating hard oblate ellipsoid monolayer: surface coverage (packing fraction) vs. reduced density (upper panel) and the equation of state (lower panel) at $\kappa=1 / 10$. The effective aspect ratio of the corresponding hard ellipse is shown in the inset of the upper panel, while the out-of-plane $(S)$ and the in-plane $(\Delta)$ order parameters are presented in the inset of the lower panel. The curves are the results of $\mathrm{PL}$ theory, while the diamond symbols correspond to $\mathrm{MC}$ simulation results. The snapshot is the result of replica exchange Monte Carlo simulation method.

not been examined here, because it is not the scope of our present study.

The principle of minimizing the intersected area with the XY plane applies also for oblate ellipsoids (see Fig. 5). The minimal intersected area, which is actually an ellipse with $\sigma_{\|, e}=\sigma_{\|}$and $\sigma_{\perp, e}=\sigma_{\perp}$ dimensions, can be achieved with ordering into the XY plane, while the outplane ordering result in higher intersected area, because $\sigma_{\|}<\sigma_{\perp}$ for oblate ellipsoids. The resulting in-plane ordering can be seen in the simulation snapshot and from the high density behavior of the effective aspect ratio $\left(\kappa_{\text {eff }} \rightarrow \kappa=\sigma_{\|} / \sigma_{\perp}\right)$. The out of plane order parameter goes to $-1 / 2$, which corresponds to complete in-plane ordering. This in-plane order is isotropic at low densities $(\Delta=0)$, while it is nematic at high densities $(\Delta>0)$. This means that the system undergoes a phase transition from a planar uniaxial nematic order $(S<0, \Delta=0)$ to a biaxial nematic one $(S<0, \Delta \neq 0)$, which can be considered as a 2D IN phase transition. The order of the uniaxial nematic-biaxial nematic (N-BN) phase transition is proved to be second order in the approximate PL theory, while the transition is higher order continuous in the simulation. This transition is the result of the competition between in-plane orientational entropy (favoring 


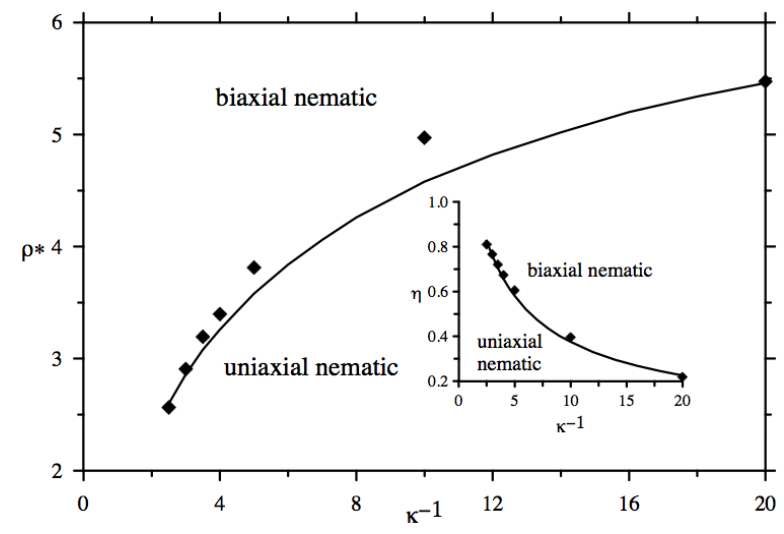

FIG. 6: Shape dependence of the uniaxial nematic-biaxial nematic (N-BN) transition of freely rotating hard oblate monolayer: reduced density vs. aspect ratio. The curves are the results of PL theory, while the diamond symbols correspond to MC simulation results. The inset shows the packing fraction of the N-BN transition as a function of aspect ratio.

disorder) and the in-plane packing entropy (favoring order). The high density structure of the hard ellipsoid monolayer resembles the nematic phase of the $2 \mathrm{D}$ hard ellipses, while at low densities the structure more or less corresponds to an isotropic phase of a polydisperse mixture of hard ellipses. One can also see that the agreement between the theory and the simulation is quite good for oblate ellipsoids, especially with respect to the equation of state. An interesting feature is that the theory overestimates the out-of-plane ordering; this could stem from the effect of higher virial coefficients, which are not included in the theory.

Fig. 6 shows the in-plane isotropic-nematic (or N-BN) transition densities as a function of aspect ratio resulting from the PL theory and simulation. On the other hand, the transition density increases with increasing shape anisotropy, while the packing fraction decreases. This is due to the fact that we go to the "volumeless" hard needle limit with decreasing $\kappa$, where the transition density saturates at finite value, while the packing fraction vanishes (note that $\kappa_{\text {eff }} \rightarrow 0$ and $\eta=\rho^{*} \pi \kappa_{\text {eff }} / 4$ ). The agreement for the transition densities is again very good between the theory and simulation. To see the effect of out-of-plane orientational freedom it is worth plotting the IN densities of 2D hard ellipse system and those of freely rotating hard oblate monolayer together (see Fig. 7). It can bee seen that the simulation does not show a substantial difference between the two systems, while the theory predicts that the out-of-plane freedom destabilizes the nematic order, i.e. the IN density curve is shifted into the direction of higher densities. In the simulation the nematic phase evolves from a very ordered planar phase (isotropic in the XY plane), where the effective aspect ratio is almost identical with the aspect ratio of the ellipsoids (see Fig. 5), i.e. the corresponding hard ellipse system is almost monodisperse. This is

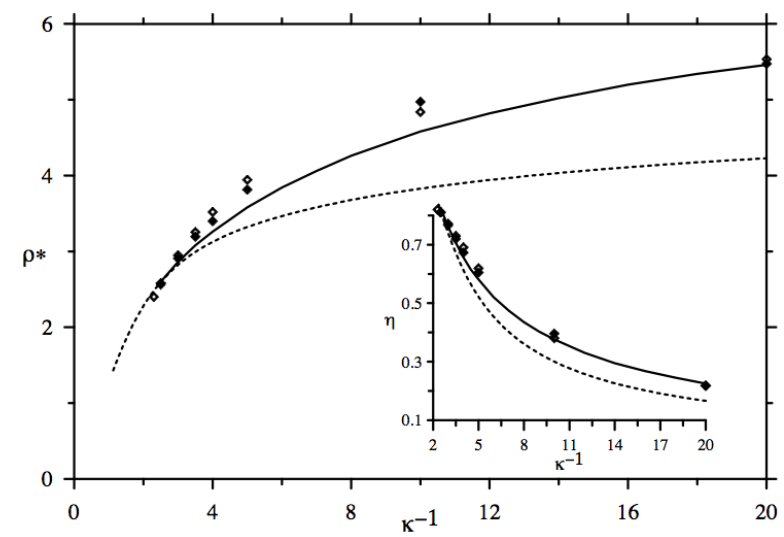

FIG. 7: Comparison of the transition densities of freely rotating hard ellipses and confined hard oblates in the reduced density-aspect ratio plane. The continuous (ellipsoids) and dashed (ellipses) curves are the results of PL theory, while the open and filled diamond symbols correspond to MC simulation results for hard ellipses and hard ellipsoids, respectively. The inset shows the packing fractions of IN and N-BN transitions as a function of the aspect ratio.

not the case in the theory, where the transition happens at such densities, where the corresponding hard ellipse system is still polydisperse with larger shape anisotropy $\left(\kappa_{\text {eff }}\right)$ than $\kappa$. As a result the effect of out-of-plane orientational freedom is more pronounced in the theory than in the simulation. This shows that the theory exaggerates the effect of out-of-plane orientational freedom on the N-BN phase transition.

Finally we show how the IN transition properties change with switching on the out of plane orientational freedom through the varying limiting polar angle $\left(\theta_{c}\right)$, which is between 0 and $\pi / 2$ for both prolate and oblate shapes. If $\theta_{c}=\pi / 2\left(\cos \theta_{c}=0\right)$ the prolate ellipsoids on the XY plane behave like the system of hard ellipses, i.e. they undergo a $2 \mathrm{D}$ IN phase transition with increasing density (see Fig. 8). However this phase transition is destabilized with decreasing the limiting angle $\left(\theta_{c}\right)$, which corresponds to increasing $\cos \theta_{c}$. As the orientational window is widened (decreasing $\theta_{c}$ ), the prolate ellipsoids tend to minimize their occupied area on the XY surface, which results in less anisotropic in-plane ellipses and higher transition densities for all studied aspect ratios. In addition to this, the in-plane order transforms continuously into out-of-plane order, i.e. $\mathrm{S}$ becomes positive with $\cos \theta_{c}$ (see Fig. 8). Hence, the $2 \mathrm{D}$ IN transitions turn into N-BN transitions for confined prolates which are lost for the free rotating case. The surface coverage (packing fraction) curves show very clearly that the occupied area on the XY surface increases enormously with decreasing the polar angle restriction, i.e. the $2 \mathrm{D}$ IN phase transition is destabilized with decreasing $\theta_{c}$. The value of $\theta_{c}$ where the orientational ordering transition is preempted by the positional one (freezing transition) cannot be determined with the present PL theory. 
Interestingly, MC simulation shows that the IN density is practically not affected by the value of limiting polar angle, but the transition disappears at values of $\theta_{c}$ close to zero. Note that the IN transition density curves move into the direction of lower densities with increasing shape anisotropy because the occupied area of the ellipsoids becomes larger with increasing $\kappa$ as $\sigma_{\|}>\sigma_{\perp}$. The case of oblate ellipsoids is different because $\theta_{c}=\pi / 2$ corresponds to the freely rotating limit, while $\theta_{c}=0$ is the hard disk limit. Starting from the freely rotating case one can see that the IN transition density shows a maximum at an intermediate value of $\theta_{c}$, which can be attributed to the combined effect of decreasing in-plane shape anisotropy and the increasing intersected area of the ellipsoids with decreasing $\theta_{c}$. The N-BN transition packing fraction behaves simply since it is always an increasing function of $\cos \theta_{c}$, i.e the nematic phase is destabilized. This means that the nematic-biaxial nematic curve and the isotropicsolid curves must cross each other at a threshold value of $\cos \theta_{c}$ making the nematic order metastable and producing a plastic solid (an orientationally disordered solid). This plastic solid would eventually turn into an ordered solid at higher densities. However, this value of $\cos \theta_{c}$ has not been searched by the simulation and theory, because our study focuses only on the orientational ordering. Finally, the $S$ order parameter shows that the system undergoes a structural change from in-plane order into outof-plane order at the N-BN transition. Our results show that the effect of out-of-plane orientational freedom is to minimize the occupied area on the surface which allows the most efficient packing of the ellipsoids on the XY plane.

\section{CONCLUSIONS}

We have examined the orientational ordering properties of the monolayer of hard ellipsoids using replica exchange Monte Carlo simulation method and the ParsonsLee density functional theory. We have found that both the shape anisotropy and the orientational restriction affect substantially the orientational order on the plane. The subtle interplay between orientational and packing entropies results in different structures and phase behaviors for prolate and oblate shaped ellipsoids. The main driving force of the ordering behavior is to minimize the intersected area between the particle and the surface to realize the close packing structure with increasing surface density. The minimal intersected area is a disk for prolate, while it is an ellipse for oblate ellipsoids. Highly packed structures can be achieved with only out-of-plane (in-plane) ordering for prolate (oblate) ellipsoids, i.e. prolate ellipsoids prefer to order along the normal of the plane, while oblate ellipsoids like to order in the plane. The monolayer of prolate ellipsoids with very small out-of-plane orientational freedom behaves almost identically to the system of $2 \mathrm{D}$ hard ellipses, i.e. it form $2 \mathrm{D}$ isotropic and nematic phases. The gradual rise of the

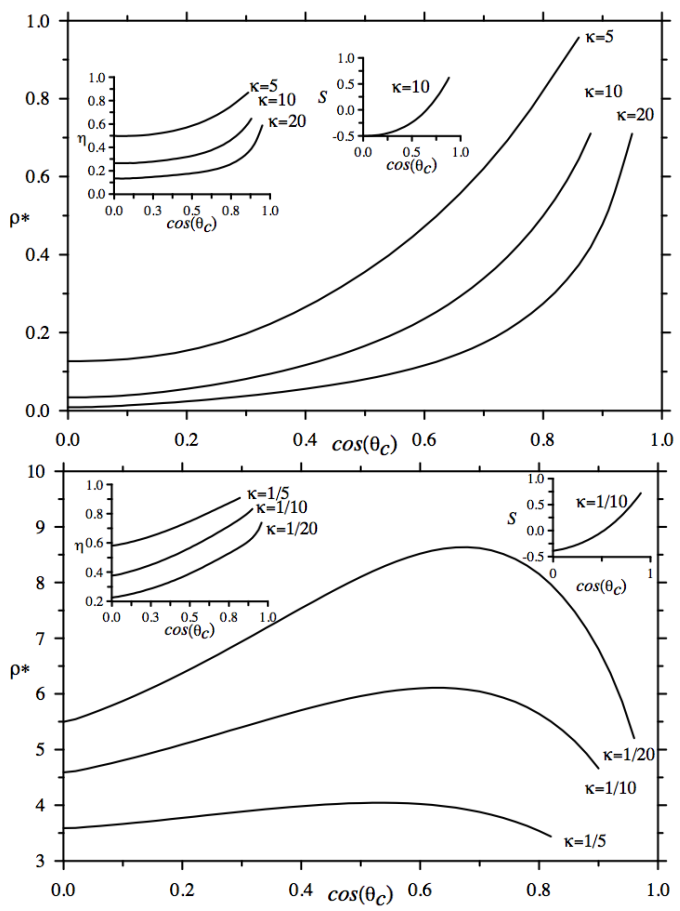

FIG. 8: Monolayers of orientationally restricted hard ellipsoids: N-BN transition density vs. $\cos \theta_{c}$. The upper panel is for prolate shapes while the lower one for oblate shapes. Insets show the $\cos \theta_{c}$ dependence of the surface coverage and the out-of-plane order parameter at the N-BN ordering transition. The curves are the results of PL theory.

out-of-plane freedom, through decreasing $\theta_{c}$, allows the ellipsoid particle to lean out from the plane and to decrease the intersected area with the plane. This involves less anisotropic shape in the interactions and destabilization of the biaxial nematic phase (2D nematic) with respect to uniaxial nematic one (2D isotropic). At full orientational freedom $(0<\theta<\pi)$ prolate ellipsoids do not form an isotropic phase, but they are ordered along the normal of the confining plane even at very low densities and behave similarly to the 2D system of hard disks at high densities.

The monolayer of oblate ellipsoids without out-of-plane freedom is identical to the $2 \mathrm{D}$ system of hard disks. The gradually increasing freedom in polar angle $(\theta)$ allows the oblate ellipsoid to decrease its intersected area with the confining plane through leaning out from the plane. This makes the ordering planar for the main symmetry axis of the ellipsoid. However the anisotropic interactions between the tilted ellipsoids give rise to an additional inplane order, i.e. the phase is biaxial nematic, which is due to the excluded area gain coming from the in-plane ordering of elliptical intersections. In the freely rotating case the oblate ellipsoids form a planar nematic phase at low densities and a biaxial nematic one at high densities. Interestingly, the IN density of $2 \mathrm{D}$ ellipses is almost identical to that of freely rotating ellipsoids confined to 
a plane. In summary the out-of-plane orientational freedom stabilizes the in-plane nematic ordering in the monolayer of oblate ellipsoids, while the opposite happens in the monolayer of prolate ellipsoids. This is congruent with the $2 \mathrm{D}$ ellipse and 2D disk phase diagrams, which are obtained as limiting cases in this study. In our simple model we have neglected the effect of out-of-plane positional freedom which we expect to have a relatively small effect on the system behavior. On the other hand, not including a soft wall-particle interaction may lead to deviations from experimental set-ups where capillary phenomena are frequently important. No doubt, the inclusion of this interaction would substantially increase the computation burden of the problem. We leave this issue for future studies.

\section{Acknowledgments}

SV acknowledges the financial support of the Hungarian State and the European Union under the TAMOP4.2.2.A-11/1/KONV-2012-0071. Grant FIS2013-47350C5-1-R from Ministerio de Educación y Ciencia of Spain is also acknowledged.
[1] S. Sacanna, D. J. Pine and Gi-Ra Yi, Soft Matter, 9, 8096 (2013).

[2] W. Qi, J. de Graaf, F. Qiao, S. Marras, L. Manna, and M. Dijkstra, Nano Lett. 12, 5299 (2012).

[3] K. J. Lee, J. Yoon and J. Lahann, Current Opinion in Colloid and Interface Science 16, 195 (2011).

[4] D. Florea and H. M. Wyss, J. Coll. Interface Sci. 416, 30 (2014).

[5] N. F. Bouxsein, C. Leal, C. S. McAllister, K. K. Ewert, Y. Li, C. E. Samuel, and C. R. Safinya, , J. Am. Chem. Soc. 133, 7585 (2011).

[6] Ravi Kumar Pujala, Nidhi Joshi, H. B. Bohidar, Colloid Polym. Sci. 3651 (2015), DOI 10.1007/s00396-015-36513.

[7] T. E. Herod and R. S. Duran, Langmuir 14, 6606 (1998).

[8] F. Kim, S. Kwan, J. Akana, and P. D. Yang, J. Am. Chem. Soc. 123, 4360 (2001).

[9] H. Razafindralambo, A. Richel, M. Paquot, L. Lins and C. Blecker, J. Phys. Chem. B 116, 3998 (2012).

[10] G. B. Davies, T. Krüger, P. V. Coveney, J. Harting and F. Bremse, Adv. Mater. 26, 6715 (2014).

[11] G. B. Davies, T. Kr $\widetilde{A} \frac{1}{4}$ ger, P. V. Coveney, J. Harting and F. Bremse, Soft Matter 10, 6742 (2014).

[12] Z. Zheng, F. Wang, and Y. Han, Phys. Rev. Lett. 107, 065702 (2011).

[13] C. K. Mishra, A. Rangarajan, and R. Ganapathy, Phys. Rev. Lett. 110, 188301 (2013).

[14] E. L. Hansen, S. Jabbari-Farouji, H. Mauroy, T. S. Plivelic, D. Bonne and J. O. Fossuma, Soft Matter 9,9999 (2013).

[15] L. S. Li and A. P. Alivisatos, Adv. Mater. 15, 408 (2003).

[16] K. Béneut, D. Constantin, P. Davidson, A. Dessombz, and C. Chanéac, Langmuir 24, 8205 (2008).

[17] D. Constantin, P. Davidson, and C. Chanéac, Langmuir 26, 4586 (2010).

[18] J. A. Cuesta and D. Frenkel, Phys. Rev. A 42, 2126 (1990).

[19] A. M. Somoza and R. C. Desai, J. Phys. Chem. 96, 1401 (1992).

[20] D. Kramer, A. Ben-Shaul, Z.-Y. Chen and W. M. Gelbart, J. Chem. Phys. 96, 2236 (1992).

[21] M. R. Fisch and C. Rosenblatt, J. Phys. II 4, 103 (1994).

[22] H. Schlacken, H.-J. Mogel, and P. Schiller, Mol. Phys. 93, 777 (1998).

[23] S. Varga and I. Szalai, Mol. Phys. 95, 515 (1998).
[24] S. Varga and I. Szalai, J. Mol. Liq. 85, 11 (2000).

[25] R. van Roij, M. Dijkstra and R. Evans, J. Chem. Phys. 113, 7689 (2000).

[26] R. van Roij, M. Dijkstra and R. Evans, Europhys. Lett. 49, 350 (2000).

[27] A. C. D. van Enter and S. B. Shlosman, Phys. Rev. Lett. 89, 285702 (2002).

[28] M. Cosentino Lagomarsino, M. Dogterom, and M. Dijkstra, J. Chem. Phys. 119, 3535 (2003).

[29] Y. Martínez-Ratón, E. Velasco, and L. Mederos, J. Chem. Phys. 122, 064903 (2005).

[30] Y. Martínez-Ratón, E. Velasco, and L. Mederos, J. Chem. Phys. 125, 014501 (2006).

[31] R. L. C. Vink, Phys. Rev. Lett. 98, 217801 (2007).

[32] Y. Martínez-Ratón, Liq. Cryst. 38, 697 (2011).

[33] Wen-Sheng Xu, Yan-Wei Li, Zhao-Yan Sun, and Li-Jia An, J. Chem. Phys. 139, 024501 (2013).

[34] Ravi Kumar Pujala and H. B. Bohidar, Phys. Rev. E 88 ,052310 (2013).

[35] G. Bautista-Carbajal and G. Odriozola, J. Chem. Phys. 140, 204502 (2014).

[36] Wen-Sheng Xu, Xiaozheng Duan, Zhao-Yan Sun, and LiJia An , J. Chem. Phys. 142, 224506 (2015).

[37] D. Frenkel and R. Eppenga, Phys. Rev. A 31, 1776 (1985).

[38] Z. Zhang, P. Pfleiderer, A. B. Schofield, C. Clasen, and J. Vermant, J. Am. Chem. Soc. 133, 392 (2011).

[39] M. González-Pinto, Y. Martínez-Ratón, E. Velasco and S. Varga, PCCP 17, 6389 (2015).

[40] Y. Martínez-Ratón, S. Varga and E. Velasco, J. Chem. Phys. 140, 204906 (2014).

[41] E. Meneses-Juarez, S. Varga, and G. Odriozola, Soft Matter 9, 5277 (2013).

[42] S. Varga, E. Meneses-Juarez, and G. Odriozola, Soft Matter 9, 11178 (2013).

[43] J. D. Parsons, Phys. Rev. A 19, 1225 (1979).

[44] S. D. Lee, J. Chem. Phys. 87, 4972 (1987) ; S. D. Lee, J. Chem. Phys. 89i, 7036 (1988).

[45] S. C. McGrother, D. C. Williamson, and G. Jackson, J. Chem. Phys. 104, 6755 (1996).

[46] P. J. Camp, C. P. Mason, M. P. Allen, A. A. Khare and D. A. Kofke, J. Chem. Phys. 105, 2837 (1996).

[47] P. Padilla and E. Velasco, J. Chem. Phys. 106, 10299 (1997).

[48] H. Reiss, H. L. Frisch, and J. L. Lebowitz, J. Chem. Phys. 
31, 369 (1959).

[49] E. Marinari and G. Parisi, Europhys. Lett. 19, 451 (1992).

[50] A. P. Lyubartsev, A. A. Martinovski, S. V. Shevkunov, and P. N. Vorontsov-Velyaminov, J. Chem. Phys. 96, 1776 (1992).

[51] K. Hukushima and K. Nemoto, J. Phys. Soc. Jpn. 65, 1604 (1996).

[52] G. Bautista-Carbajal, A. Moncho-Jordá and G. Odrio- zola, J. Chem. Phys. 138, 064501 (2013).

[53] G. Odriozola, J. Chem. Phys. 131, 144107 (2009).

[54] T. Okabe, M. Kawata, Y. Okamoto and M. Mikami, Chem. Phys. Lett. 335, 435 (2001).

[55] A. Donev, F. H. Stillinger, P. M. Chaikin S. and Torquato, Phys. Rev. Lett. 92, 255506 (2004).

[56] A. Donev, S. Torquato and F. H. Stillinger, J. Comp. Phys. 202, 737 (2005). 